\section{Stockley's Herbal Medicines Interactions: A Guide to the Interactions of Herbal Medicines}

Williamson E, Driver S, Baxter K, editors. Pharmaceutical Press, London, United Kingdom, 2009. Hardcover, 423 pages. ISBN 978085369760 2. \$89.99.

Stockley's Herbal Medicines Interactions is a 423-page guide to the interactions of more than 150 herbal medicines with conventional medicines. The term "herbal medicines" in the title is widely defined to include products from plant sources as well as other types of dietary supplements and nutraceuticals (e.g., acidophilus, beta-carotene, and coenzyme Q10), which in Canada would all be classified as natural health products. The introduction to the book includes a brief overview of patterns of use and rates of reported adverse events or interactions related to herbal medicines in a range of patient groups, including hospital inpatients, children, and elderly people. Perhaps the most useful part of the introduction is the 2 tables listing drugs and herbal medicines that affect the cytochrome P450 isoenzymes CYP1A2 and CYP3A4, respectively, classified as inducers, substrates, and inhibitors.

Most of the book consists of the monographs for each herbal medicine, which include synonyms, key constituents, indications, and pharmacokinetics. The detailed analysis of how each product may interact with specific pharmaceuticals or classes of pharmaceuticals, foods, and other herbal products is fully referenced and clearly indicates the type of evidence upon which the suspected interactions are based (e.g., clinical evidence or experimental laboratory evidence). Purported mechanisms of action are discussed, and a section called "Importance and Management" represents an attempt to explain the clinical significance of the interaction, which I found to be both balanced and useful for practice-based decision-making.
Each interaction is rated according to 3 categories: action (whether or not any action needs to be taken to address the interaction), severity (the likely effect on a patient of an unmanaged interaction), and evidence (the weight of the available evidence). In addition, each interaction is categorized (on the basis of a combination of these ratings) using 1 of 5 symbols, whose meanings range from "may have a life-threatening outcome, so avoid this combination" to "not considered to be of clinical significance". The symbols themselves are relatively intuitive and provide a quick reference for readers with limited time. Finally, there is a full index, which allows the reader to find both drugs and herbal medicines listed under a range of common names.

As with all books on this topic, the text is limited by the lack of data available for some products. For example, the Ginkgo entry is 12 pages long and reviews suspected interactions with 28 other types of products, but the Hoodia review is a single page with the simple statement that "no interactions were found." Because the book is organized by botanical products, it is a bit cumbersome to access information about specific drugs. For example, preparing a talk about interactions between herbal medicines and warfarin would require consulting more than 30 different pages in the book (all listed in the index). This book is probably best suited to answering questions about specific herbal-drug combinations that a patient may be taking (or may be considering taking).

Overall, I found this book to be comprehensive, evidencebased, and useful for clinicans. Of the books currently available on this topic, it is definitely one of the best.

Heather Boon, BScPhm, PhD

Leslie Dan Faculty of Pharmacy

University of Toronto

Toronto, Ontario 